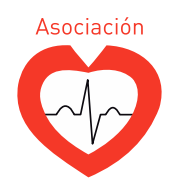

SOCIEDAD COLOMBIANA DE CARDIOLOGÍA \& CIRUGÍA CARDIOVASCULAR

\section{Revista Colombiana de \\ Cardiología \\ www.elsevier.es/revcolcar}

CARDIOLOGÍA DEL ADULTO - REVISIÓN DE TEMAS

\title{
Coronavirus - COVID 19: Más allá de la enfermedad pulmonar, qué es y qué sabemos del vínculo con el sistema cardiovascular
}

\author{
Juan David López-Ponce de León ${ }^{\mathrm{a}, \mathrm{b}, *}$, Paula Andrea Cárdenas-Marín ${ }^{\mathrm{b}, \mathrm{c}}$, \\ Germán Camilo Giraldo-González ${ }^{\mathrm{b}, \mathrm{d}}$ y Álvaro Herrera-Escandón ${ }^{\mathrm{b}, \mathrm{e}, \mathrm{f}}$
}

\author{
a Servicio de Cardiología, Unidad de Insuficiencia Cardíaca y Trasplante. Fundación Valle del Lili, Cali, Colombia \\ b Universidad ICESI, Cali, Colombia \\ c Medicina Interna, ICESI-Fundación Valle del Lili, Cali, Colombia \\ d Departamento de Investigaciones Clínicas, Cali, Colombia \\ e Universidad del Valle, Cali, Colombia \\ f Clínica Neurocardiovascular DIME, Cali, Colombia
}

Recibido el 6 de abril de 2020; aceptado el 8 de abril de 2020

Disponible en Internet el 23 de abril de 2020

\section{PALABRAS CLAVE \\ Falla cardiaca; \\ Coronavirus; \\ Miocarditis; \\ Lesión miocárdica; \\ Distrés respiratorio \\ agudo del adulto}

\section{KEYWORDS}

Heart failure;

Coronavirus;

Myocarditis;

\begin{abstract}
Resumen La infección por SARS-CoV2 es una pandemia. Se creía que el primer caso de esta enfermedad ocurrió el 8 de diciembre de 2019 en la provincia de Hubei en China, aunque posteriormente se indicó que el primer caso confirmado por laboratorio ocurrió el $1 .^{\circ}$ de diciembre de 2019 ante la presencia de un brote de neumonía en 59 pacientes sospechosos en un mercado local de mariscos en Wuhan. No solo produce patología respiratoria, con frecuencia compremete el sistema cardiovascular ya que produce lesión miocárdica, miocarditis, y, con cierta frecuencia, aumenta la descompensación de enfermedades cardiovasculares preestablecidas. En este artículo se trata de dilucidar el componente cardiovascular hasta ahora existente en la literatura y se sugieren algunos pasos a seguir en pacientes con estas enfermedades, acorde con la evidencia actual.

(C) 2020 Sociedad Colombiana de Cardiología y Cirugía Cardiovascular. Publicado por Elsevier España, S.L.U. Este es un artículo Open Access bajo la licencia CC BY-NC-ND (http:// creativecommons.org/licenses/by-nc-nd/4.0/).
\end{abstract}

COVID-19 coronavirus: More than just a lung disease: what it is and what we know about the link with the cardiovascular system

Abstract Infection due to SARS-CoV2 is a pandemic. It is believed that the first case occurred on 8 December 2019 in Hubei province in China, although it was later indicated that the first

\footnotetext{
* Autor para correspondencia.

Correo electrónico: jd1lopez@gmail.com (J.D. López-Ponce de León).
} 
Myocardial lesion; Adult acute respiratory disease laboratory-confirmed case occurred on 1 December 2019 due to the presence of an outbreak of suspected pneumonia in 59 patients in a shellfish market in Wuhan. It not only caused a respiratory disease, it often compromised the cardiovascular system since it produces a myocardial lesion, myocarditis, and, less often, increased the decompensation of pre-established cardiovascular diseases. An attempt is made in this article to elucidate the cardiovascular component presented in the current literature, and to suggest some steps to follow in patients with these diseases in accordance with the current evidence.

(c) 2020 Sociedad Colombiana de Cardiología y Cirugía Cardiovascular. Published by Elsevier España, S.L.U. This is an open access article under the CC BY-NC-ND license (http:// creativecommons.org/licenses/by-nc-nd/4.0/).

\section{Introducción}

La infección por SARS-CoV2 es una pandemia. Se creía que el primer caso de esta enfermedad ocurrió el 8 de diciembre de 2019 en la provincia de Hubei en China ${ }^{1}$, pero, posteriormente, se indicó que el primer caso confirmado por laboratorio ocurrió el $1 .^{\circ}$ de diciembre de 2019 , ante la presencia de un brote de neumonía en 59 pacientes sospechosos en un mercado local de mariscos en Wuhan, por lo cual se lanzó una alerta epidemiológica con el aislamiento de un nuevo coronavirus en el tracto respiratorio inferior, denominado inicialmente 2019-nCoV². El 7 de enero de 2020 fue identificado como agente causal del brote un nuevo tipo de virus de la familia Coronaviridae denominado SARS-CoV2 y el 11 de marzo la Organización Mundial de la Salud declaró la pandemia ${ }^{3}$. Al día 4 de abril del año en curso, se reportaban 1'159.515 casos en más de 200 países, y 62.376 muertes en todo el mundo ${ }^{4}$, especialmente de adultos mayores con comorbilidades, como hipertensión, diabetes y enfermedad cardiovascular, ocasionando así un gran impacto social y económico ${ }^{5,6}$.

La principal manifestación de esta enfermedad es el compromiso respiratorio, el cual puede ocasionar desde síntomas respiratorios leves, hasta síndrome de dificultad respiratoria del adulto con desenlaces potencialmente fatales ${ }^{7}$. Se ha descrito que la enfermedad cardiovascular preexistente y otros factores de riesgo cardiovascular pueden generar mayor susceptibilidad a infección por COVID-19 y un escenario con mayores complicaciones clínicas. Además, se ha encontrado que esta infección puede asociarse con complicaciones cardiacas de novo, como lesión miocárdica aguda en cerca del 8 al $12 \%$ de los pacientes, miocarditis y arritmias en el $16,7 \%$, cer los mecanismos fisiopatológicos que están relacionados con el compromiso cardiovascular en la infección por SARSCoV2-COVID-19, cómo sospecharla, cómo evaluarla y qué intervenciones terapéuticas tener en cuenta en el tratamiento de este grupo de pacientes.

\section{Virus SARS-CoV2 e infección por COVID 19}

El SARS-CoV2 es un virus ARN monocatenario del género beta-coronavirus, familia Coronaviridae. Se conocen siete especies de esta familia que pueden causar infecciones en
Tabla 1 Características biológicas del SARS-CoV2

- Estructura del virus y características de la infección

- Alta tasa de transmisión

- Período de incubación prolongado

- Portadores asintomáticos o con síntomas leves

- Progresión a SDRA* y muerte

- Diseminación viral después del alivio de los síntomas

- Transmisión ambiental y fómites

SDRA: síndrome de dificultad respiratoria del adulto.

humanos, de las cuales cuatro causan principalmente síntomas respiratorios leves y tres pueden desencadenar una enfermedad potencialmente fatal, como el síndrome respiratorio agudo grave (SARS), el síndrome respiratorio del Oriente medio (MERS) y la actual COVID-19 (tabla 1) ${ }^{7}$.

La enfermedad por coronavirus presenta características inherentes que le otorgan su alto potencial de infección, transmisibilidad y desenlaces desfavorables ${ }^{8}$ ocasionando una pandemia de alto impacto mundial, como son:

- Estructura del virus y características de la infección: se deriva probablemente de un coronavirus de murciélago, cuyo reservorio principal podrían ser las serpientes de la zona de Wuhan. Se ha estudiado también la posibilidad de que su transmisión sea a través de una sopa de murciélago que se consume en la zona; además, se ha encontrado coronavirus en pangolines salvajes cuya secuencia genética coincide en un $91 \%$ con el virus causante del COVID-19 ${ }^{9}$.El SARS-CoV2 penetra en la célula huésped a través de la interacción entre su glucoproteína espiga (S) y una proteína de membrana plasmática, el receptor de la enzima convertidora de angiotensina 2 (ECA2), una aminopeptidasa unida a la membrana con un papel vital en los sistemas cardiovascular e inmune ${ }^{10}$. El virus invade las células epiteliales alveolares comprometiendo el sistema respiratorio y causando un cuadro clínico de mayor severidad en pacientes con enfermedad cardiovascular, lo que podría estar asociado con mayor secreción de ECA2 en estos pacientes. Adicionalmente, puede desarrollar linfopenia ${ }^{11}$, sobre todo en pacientes con enfermedad grave, que puede estar acompañada de inmunosupresión, lo cual los predispone a coinfec- 
ciones graves, especialmente bacterianas ${ }^{12}$. Otro aspecto importante es la posibilidad de recombinación activa de SARS-CoV2 lo que puede generar variantes del virus dentro del huésped. La presencia de estos haplotipos heterogéneos del virus, así como su interacción con las características genéticas y epigenéticas del huésped pueden hacer difícil predecir el curso de la enfermedad; esto podría explicar las diferencias en el compromiso clínico y la tasa de letalidad -entre otros factores - en países como China e Italia, secundario a una selección natural de grupos virales más virulentos en ciertas zonas o un comportamiento más agresivo del virus debido a factores genéticos, clínicos o medioambientales, como la edad avanzada, la prevalencia de comorbilidades o las condiciones climáticas ${ }^{13}$.

- Alta tasa de transmisión: para el SARS-CoV2 el R0, o número de reproducción básica, es decir, la velocidad con la que una enfermedad puede propagarse en una población, se ha estimado en 2,4 . Se han encontrado valores de 2,2 en China y 2,8 en Italia, similares a los hallados en la infección por SARS $(2,0$ a 5,0$)$ y MERS $(2,7$ a 3,9$)$, lo que sugiere una propagación rápida y sostenida ${ }^{14-16}$.

- Período de incubación prolongado: aunque el RO del SARS-CoV2 es similar al del SARS y al del MERS, existen características específicas que pueden explicar una propagación viral mucho mayor, como lo son el período de incubación de 6,4 días (2,1-11,1 días), comparable con el del MERS (4-7 días) y un poco más largo que el del SARS (3-5 días) ${ }^{17}$.

- Pacientes asintomáticos o con síntomas leves: se ha encontrado aislamiento de SARS-CoV2 en garganta y nariz días antes de los síntomas y se han documentado cargas virales positivas similares a las de pacientes enfermos, en pacientes asintomáticos o con síntomas leves. En un estudio realizado en población japonesa en Wuhan se describe una razón de asintomáticos de 30,8\% (IC 95\% $7,7-53,8 \%)^{18}$. Por su parte, en otro estudio realizado en los pasajeros del crucero Diamond Princess se encontró que la población asintomática de casos de COVID-19 podía ser hasta del 17,9\% (IC 95\% 15,5-20,2\%) ${ }^{19}$. Un mecanismo atribuible es la baja prevalencia de síntomas del tracto respiratorio superior, menos del $20 \%$, en cuyo caso los receptores ECA2 son mínimos. Esto ilustra cómo los pacientes pueden ser portadores o manifestar síntomas leves que pueden contribuir a la transmisión del virus.

- Progresión desfavorable hacia un síndrome de dificultad respiratoria del adulto y muerte: en reportes de literatura en China, se ha encontrado que en un espectro clínico de la enfermedad predominantemente leve en la mayoría de los casos (80\%), hasta el $15 \%$ de los pacientes puede progresar a enfermedad grave y un $5 \%$ requerir unidad de cuidados intensivos ${ }^{20}$. Sin embargo, estos datos pueden variar según las características de la población, como se ha visto en Italia o España ${ }^{21}$.

- Diseminación viral después del alivio de los síntomas: existe la posibilidad de transmisión del virus después de la desaparición de los síntomas. Se ha detectado ARN del SARS-CoV2 en heces varios días después de la enfermedad del paciente o durante la convalecencia; además, se ha encontrado eliminación viral prolongada en el esputo después del cese sintomático ${ }^{21}$.
Tabla 2 Mecanismos de lesión cardiaca en infección por SARS-CoV2

- Lesión miocárdica directa

- Inflamación sistémica

- Infarto agudo de miocardio tipo 1

- Infarto agudo de miocardio tipo 2

- Efectos adversos de terapias farmacológicas

- Alteraciones electrolíticas

- Transmisión ambiental y fómites: se ha encontrado que la vida media del SARS-CoV2 puede ser de 1.2 horas en gotas (IC 95\%, 0.64 - 2.64 horas) ${ }^{29}, 3$ horas en aerosol, hasta 24 horas en cartón y hasta 72 horas en plástico y acero inoxidable; esto debe llevar a adoptar medidas estrictas de higiene de manos y manejo de fómites ${ }^{22}$.

\section{Epidemiología}

La infección por SARS-CoV2 - COVID-19 actualmente afecta a cerca de 200 países, con más de 10 millones de casos, cerca de 5.664.335 pacientes recuperados y más de 508.077 muertes. En el momento, el país más afectado en cuanto a número de casos es Estados Unidos, con cerca de 2.681.811 casos y mortalidad con cerca de 128.783 muertes. El primer país de Latinoamérica afectado fue Brasil, donde el primer caso se reportó el 26 de febrero, mientras que la primera muerte se registró en Argentina el 7 de marzo. En el momento, Brasil es el país latinoamericano con el mayor número de casos, con cerca de 1.370 .488 y 58.385 muertes. En Colombia el Ministerio de Salud y Protección Social reportó el primer caso de COVID-19 el 6 de marzo de 2020 en una ciudadana proveniente de Milán, Italia, y el primer fallecido se reportó el 13 de marzo y se trató de un taxista que transportó a turistas italianos el 4 de marzo. A día de hoy hay cerca de 95.043 casos reportados y 3.223 muertes.

\section{Consideraciones fisiopatológicas}

Además de la interacción del virus con el receptor de ECA2, cuyo principal rol en el pulmón hace que sea el sistema más frecuentemente afectado, existe una alta incidencia de compromiso cardiovascular en estos pacientes, por lo cual se han inferido varios mecanismos fisiopatológicos (tabla 2):

1. Lesión miocárdica directa: el SARS-CoV2 ingresa a las células del huésped por la unión de la proteína espiga a la ECA2 la cual es expresada en el corazón y desempeña un papel preponderante en la homeostasis cardiovascular por medio del sistema renina-angiotensina-aldosterona (SRAA) generando directamente lesión miocárdica ${ }^{10}$.

2. Inflamación sistémica: el COVID-19, en su forma más severa, ocasiona una respuesta inflamatoria sistémica aguda, subsecuente a la liberación de citoquinas proinflamatorias las cuales pueden causar daño y falla multiorgánica, comprometiendo el sistema cardiovascular ${ }^{2,23}$.

3. Infarto agudo de miocardio tipo 1: la respuesta inflamatoria sistémica puede ocasionar un incremento del estrés en la circulación coronaria lo que puede llevar a una ruptura de placa e infarto agudo de miocardio. 
4. Infarto agudo de miocardio tipo 2: el síndrome de respuesta inflamatoria sistémica, con el consecuente incremento de la demanda de oxígeno asociado a la hipoxia por el compromiso pulmonar, genera un incremento en la demanda miocárdica de oxígeno que no se suple, y causa lesión miocárdica.

5. Efectos adversos de terapias farmacológicas: los medicamentos antivirales, antimaláricos y esteroides usados para el tratamiento del COVID-19 pueden generar lesión miocárdica o trastornos de la conducción, como prolongación del intervalo QT y arritmias tipo torsade de pointes $^{24}$.

6. Alteraciones en los electrolitos: el compromiso multisistémico puede generar disbalance, siendo más común la hipocalemia por la interacción del virus con el SRAA, lo cual favorece principalmente arritmias ${ }^{25}$.

\section{Cuadro clínico}

El SARS-CoV2 afecta predominantemente el sistema respiratorio y el $97,5 \%$ de los pacientes experimentan síntomas a los 11,5 días después de la exposición ${ }^{26}$. Varios autores reportan que el síntoma más frecuente es la fiebre en un $98,6 \%$ de los pacientes, seguido por fatiga (astenia, adinamia) en un $69,6 \%$ y tos seca $(59,4 \%)$. La disnea se presentó en un $32,2 \%$ de los pacientes, con mayor frecuencia en los pacientes que ingresaron a unidad de cuidados intensivos (UCI) $(63,9$ vs. $19,6 \%)$. Las comorbilidades más encontradas fueron hipertensión arterial $(31,2 \%)$, enfermedad cardiovascular (14,5\%) y diabetes (10,1\%). De los pacientes que ingresaron a $\mathrm{UCl}$ el $72,2 \%$ tenían alguna comorbilidad; el $58,3 \%$ eran hipertensos, el $25 \%$ tenían enfermedad cardiovascular y el $22,2 \%$ eran diabéticos. En esta cohorte el $26 \%$ de los pacientes requirió hospitalización en $\mathrm{UCl}$, siendo el síndrome de dificultad respiratoria $(19,6 \%)$ la complicación más frecuente, seguida por arritmias $(16,7 \%)$, choque $(8,7 \%)$ y lesión cardiaca aguda $(7,2 \%)^{6}$.

\section{Lesión miocárdica}

Está dada por la elevación de enzimas cardiacas, especialmente troponina de alta sensibilidad, o por alteraciones nuevas en el electrocardiograma o el ecocardiograma.

La lesión miocárdica aguda es la complicación cardiovascular más descrita, 7.2-28\%. Puede estar asociada a etiología no isquémica, como miocardiopatía inflamatoria aguda o síndrome de takotsubo, e isquémica con infarto tipos $1 \mathrm{y}$ 2.

Se ha reportado una asociación entre la lesión cardiaca y la mortalidad en pacientes hospitalizados en una cohorte de 416 pacientes en Wuhan. La lesión miocárdica fue definida como niveles de biomarcadores cardiacos por encima del percentil 99 para su valor de referencia y nuevas anormalidades en el ecocardiograma o el electrocardiograma. Los pacientes que la desarrollaron eran mayores (74 vs. 60 años) y tenían más comorbilidades, como hipertensión arterial $(59,8 \%)$, diabetes $(24,4 \%)$ y enfermedad coronaria $(29,3 \%)$. En los estudios adicionales, llama la atención que tenían mayores niveles de leucocitos, plaquetas, PCR y procalcitonina. Estos pacientes requirieron mayor ventilación mecánica no invasiva $(46,3 \%$ vs. $3,9 \%)$ y ventilación mecánica invasiva ( $22 \%$ vs. $4,2 \%)$ y recibieron con más frecuencia tratamiento con corticoides, inmunoglobulina y antibióticos; la complicación más común fue la falla renal aguda. El riesgo de morir para pacientes con lesión miocárdica era 4,26 veces el encontrado en pacientes sin lesión miocárdica al inicio de los síntomas (HR 4,26 IC 95\%, 1,92-9,49) y entre la admisión y el desenlace (HR 3,41 IC 95\%, 1,62-7,16). Se registró mayor mortalidad en los pacientes con lesión miocárdica $(51,2 \%$ vs. $4,5 \%)^{27}$.

\section{Miocarditis y miocardiopatía}

Hasta el momento no hay diagnóstico histológico con biopsias endomiocárdicas. Se han descrito dos reportes de casos. El primero correspondió a un paciente de 37 años, residente en China, admitido por disnea, dolor torácico, hipotensión y cardiomegalia, cuyo electrocardiograma presentaba elevación del ST inferior. Fue llevado a laboratorio de hemodinamia sin lesiones obstructivas epicárdicas, troponina marcadamente elevada $>10.000 \mathrm{ng} / \mathrm{L}$, CKMB $112,9 \mathrm{ng} / \mathrm{L}$ y BNP $21.025 \mathrm{ng} / \mathrm{L}$. El ecocardiograma reportó corazón aumentado de tamaño, función ventricular severamente disminuida, $27 \%$, y derrame pericárdico de $2 \mathrm{~mm}$. El panel viral respiratorio para coronavirus fue positivo. Fue tratado con metilprednisolona $200 \mathrm{mg} /$ día por 4 días, inmunoglobulina $20 \mathrm{~g}$ /día por 4 días, norepinefrina e inotropia con milrinone y diurético para el choque; además, se usó piperacilina/tazobactam para manejo infeccioso pulmonar. Posteriormente, se realizó ecocardiograma en el que se evidenció función ventricular en $66 \%$, con tamaño y función normal ${ }^{28}$.

El otro caso se trató de una mujer de 47 años, residente en Londres, quien consultó por disnea y dolor torácico, tos y fiebre; presentaba, además, taquicardia sinusal e hipotensión. El electrocardiograma mostró elevación del ST inferolateral, con arterias coronarias sanas. Se realizó ecocardiograma en el que se documentó derrame pericárdico de $2 \mathrm{~cm}$, con signos de taponamiento cardiaco, por lo cual se procedió a realizar pericardiocentesis guiada por ultrasonido, en la que se drenaron $540 \mathrm{ml}$ de líquido sanguinolento, el cual dio negativo para COVID-19. El hisopado nasofaríngeo fue negativo para patógenos respiratorios, excepto para COVID-19, lo que hace importante considerar un taponamiento cardiaco como causa de deterioro en un paciente con infección por SARS-CoV2 ${ }^{29}$.

Tian et al. realizaron biopsia de corazón a dos pacientes fallecidos por COVID-19 y encontraron edema, fibrosis intersticial e hipertrofia sin infiltración celular inflamatoria ${ }^{30}$.

\section{Arritmias}

Las arritmias (7-17\%) han sido descritas como una manifestación cardiovascular frecuente en pacientes con infección por COVID-19. Se ha descrito fibrilación auricular, taquicardia supraventricular paroxística, taquicardia ventricular, fibrilación ventricular y arresto cardiaco. En una cohorte de 137 pacientes se encontró que las palpitaciones estuvieron presentes en el 7,3\% de los pacientes durante la admisión ${ }^{30}$. En una cohorte china de 138 pacientes hospitalizados se encontró que el $16,7 \%$ presentó alguna arritmia cardiaca; además, 


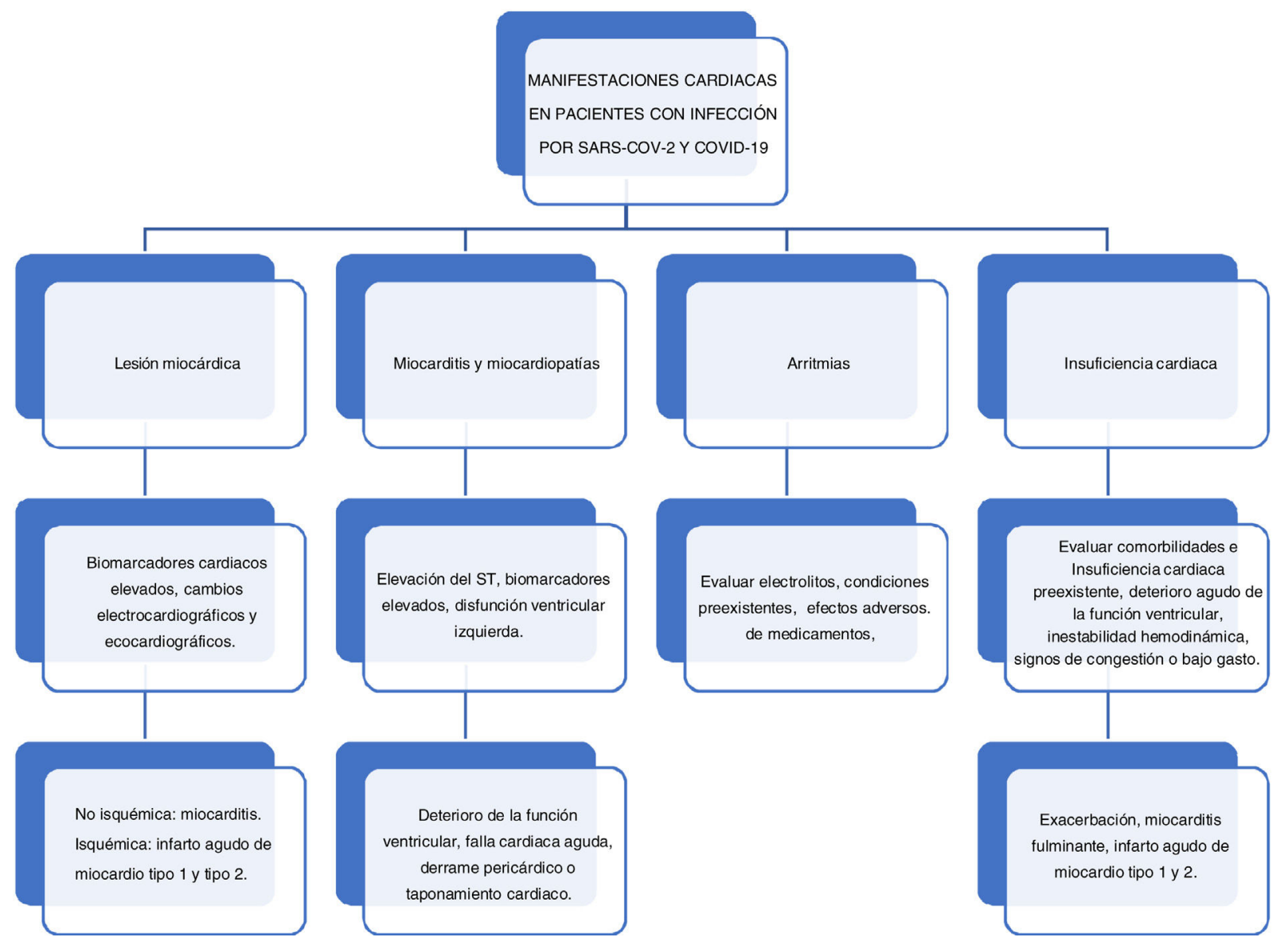

Figura 1 Manifestaciones cardíacas de los pacientes con infección por SARS-CoV2 - COVID-19.

fue más frecuente en pacientes hospitalizados en $\mathrm{UCI}(44,4$ vs. $6,9 \%)^{6}$, posiblemente con vías multifactoriales, como trastornos hidroelectrolíticos, hipoxia y estrés inflamatorio, y como efectos adversos de los medicamentos utilizados.

\section{Insuficiencia cardíaca}

La insuficiencia cardiaca puede estar asociada con antecedente de insuficiencia cardiaca crónica. En una cohorte de 416 pacientes en China, se identificó que el $4,1 \%$ de los pacientes presentaba antecedente de insuficiencia cardíaca crónica. Así mismo, en quienes se documentó lesión miocárdica, con frecuencia se hallaba antecedente de insuficiencia cardiaca $(14,6$ vs. $1,5 \%)$ y niveles más elevados de NT-proBNP $(689 \text { vs. } 139)^{27}$.

Por otro lado, en una cohorte multicéntrica en China en la que se incluyeron 191 pacientes, el 23\% tuvo insuficiencia cardiaca y de éstos el $63 \%$ no sobrevivió ${ }^{23}$. La insuficiencia cardiaca puede presentarse como una exacerbación de una insuficiencia cardiaca preexistente o secundaria a insuficiencia cardiaca aguda de reciente inicio, como un evento de miocarditis fulminante o un evento coronario agudo isquémico o hemodinámico (fig. 1).

\section{Consecuencias cardiovasculares a largo plazo}

Estudios de seguimiento a pacientes recuperados de infección por SARS-CoV han encontrado que estos pacientes presentan una alteración en el metabolismo de carbohidratos y lípidos; en un seguimiento de 25 años el 68\% tenía hiperlipidemia, el 60\% tenía trastornos del metabolismo de la glucosa y el $44 \%$ tenía anormalidades cardiovasculares. Se desconoce el mecanismo exacto, pero se encontró que en estos pacientes las concentraciones séricas de ácidos grasos libres, lisofosfatidilcolina, lisofosfatidiletanolamina y fosfatidilglicerol fueron mayores en los pacientes con antecedente de infección por SARS-CoV ${ }^{31}$, por lo cual, dada la similitud del SARS-CoV2 en su estructura con el SARS-CoV, se infiere que el nuevo virus también podría causar un daño cardiovascular a largo plazo, así que debe evaluarse esta posibilidad en este grupo de pacientes (fig. 2).

\section{Recomendaciones para el paciente con insuficiencia cardiaca, miocarditis aguda fulminante y trasplante cardiaco con sospecha o confirmación de infección por coronavirus SARS-CoV2}

Es necesario resaltar que es fundamental interrogar y aclarar si el paciente que ingresa a la institución pertenece a alguno de los grupos o casos posibles para COVID-19 y enfocar su patología de acuerdo con los lineamientos de la guía institucional.

En vista de que los síntomas respiratorios son solo parte del espectro clínico y el riesgo de exposición del personal 


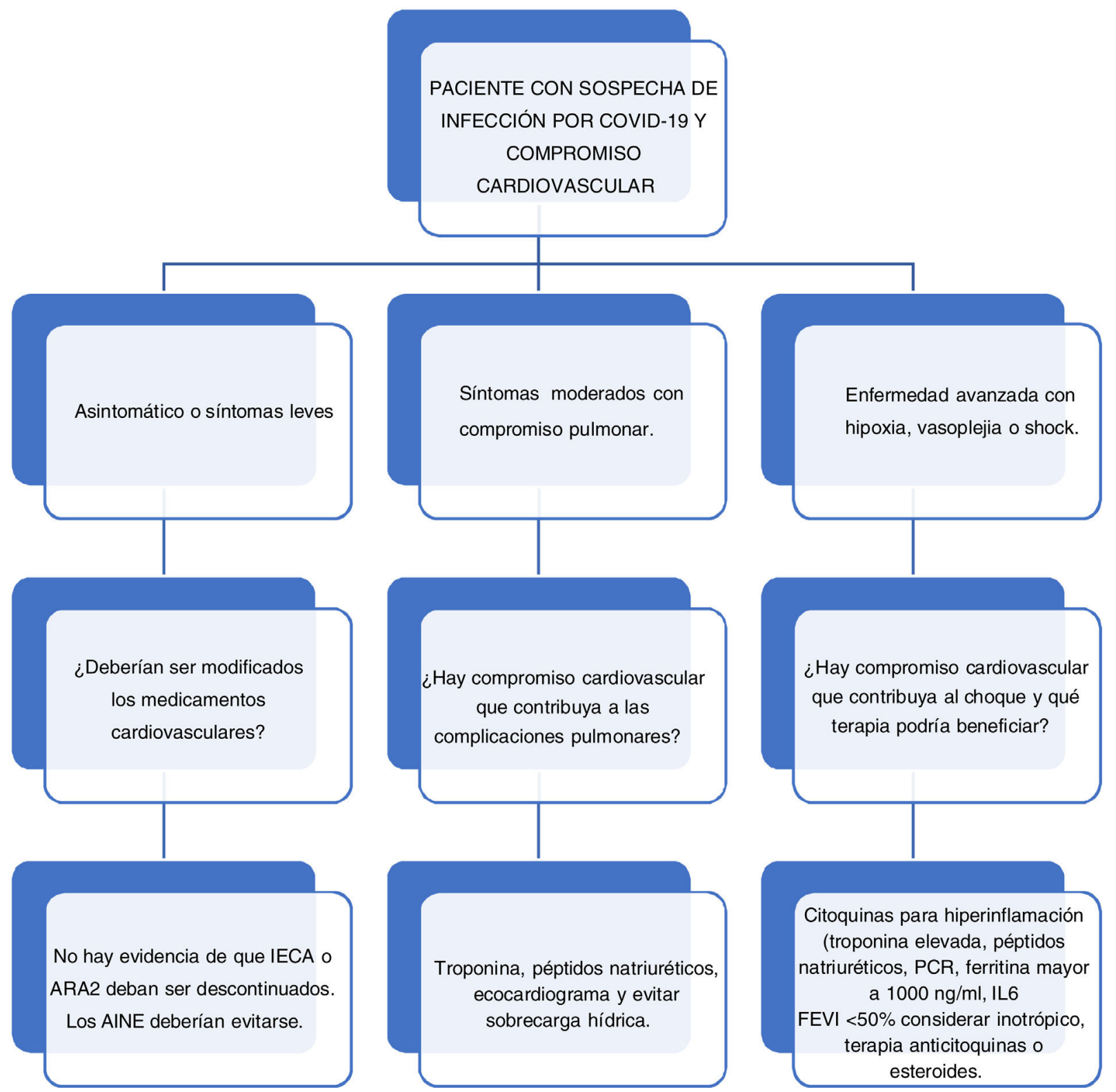

Figura 2 Flujograma de intervención en pacientes con infección por COVID-19 y sospecha de compromiso cardiovascular. Ajustado de: COVID-19 Ilness and Heart Failure. JAMA. 2020.

de salud es alto, se sugiere considerar también como sospechosos a aquellos que debuten con las siguientes manifestaciones sin una etiología en riesgo identificado.

- Shock cardiogénico.

- Síndrome coronario agudo.

- Miocardiopatía por estrés.

- Descompensación o debut de insuficiencia cardiaca.

\section{Pacientes con antecedente de insuficiencia cardiaca en el contexto actual de pandemia por COVID-19}

La enfermedad cardiovascular, como se ha descrito previamente, constituye un factor de riesgo importante para la infección por SARS-CoV2 y sus potenciales complicaciones y mortalidad; por lo tanto, este grupo de pacientes se considera un grupo de riesgo y se deben tener adoptar estrictas de prevención, como limitar las visitas médicas, hacer consultas de seguimiento por telemedicina o vía telefónica y dar las indicaciones claras de signos de descompensación, para lo cual deben consultar al servicio de urgencias.

No se recomienda la suspensión de medicamentos como IECA o antagonistas del receptor de angiotensina 2 (ARA2) dado que, si bien se ha sugerido que debido a su mecanismo de acción sobre la ECA 2 podrían aumentar el riesgo, no hay evidencia suficiente para desaconsejarlos, y, por el contrario, suspenderlos podría causar descompensación de su insuficiencia cardiaca e ir en detrimento de su supervivencia (tabla 3). 
Tabla 3 Recomendaciones para pacientes con antecedente de insuficiencia cardiaca

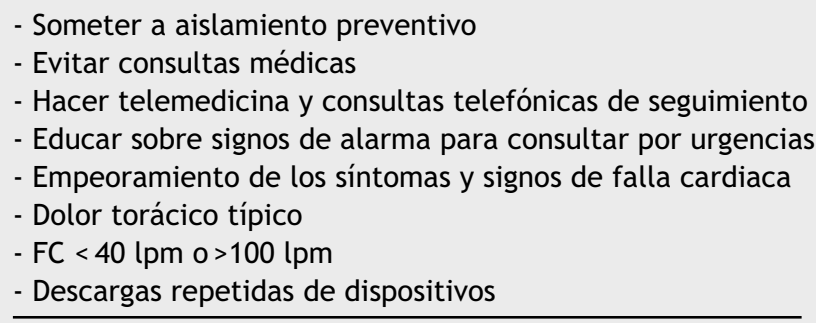

\section{Paciente en el contexto de insuficiencia cardiaca descompensada}

En los pacientes que consultan por insuficiencia cardiaca como causa de una descompensación de su patología de base, debe evaluarse la condición clínica y definir el área donde se debe hospitalizar para prevenir la exposición a infección por SARS-CoV2. Se deben identificar de forma precoz los factores de riesgo cardiovascular en todos los pacientes dado que tienen mayor riesgo de infección y deterioro.

El manejo de estos pacientes debe realizarse según las guías de insuficiencia cardiaca aguda, basado en el perfil hemodinámico para definir el requerimiento de manejo con diurético, vasodilatador o inotrópico según sea el caso. Además, se debe evaluar la causa de su descompensación para definir si el paciente requiere una intervención adicional, como un cateterismo cardiaco.

\section{Paciente en el contexto de insuficiencia cardiaca descompensada y sospecha/infección por COVID-19}

En los pacientes que consultan por insuficiencia cardiaca es preciso evaluar si se debe a una exacerbación de su patología o si corresponde a un evento agudo, el cual podría estar en relación con un evento coronario o una miocarditis viral. Deben evaluarse los síntomas, hacer un examen clínico detallado y realizar un diagnóstico diferencial con infección por SARS-CoV2 (tabla 4). Además, en cuanto al manejo farmacológico, se deben tener en cuenta las posibles repercusiones cardiovasculares (tabla 5).

\section{Pacientes con miocarditis aguda fulminante}

Las recomendaciones en los pacientes con miocarditis aguda fulminante, con base en una recopilación de datos (que son pocos) en la literatura, serían las siguientes:

1. Realizar ecocardiograma y descartar enfermedad coronaria subyacente (patrón ACS like).

2. Iniciar soporte inotrópico (milrinona, levosimendán, los pacientes presentan con frecuencia taquicardia sinusal), o asistencia ventricular necesaria para mantener la perfusión de otros órganos.

3. Descartar infección por otros virus; considerar realizar panel viral. Sin embargo, puede existir coinfección con SARS-CoV2 y otros virus respiratorios, o dar serologías y/o antigenemia positiva para virus como el dengue (casos descritos). Se debe esperar la PCR tomada de la mejor calidad para definir la probabilidad del caso.

4. Dar tratamiento viral específico avalado para el momento.

\section{Importante}

La presencia de transmisión local hace que se sospeche la posibilidad de infección por SARS-CoV2 en algunos pacientes sintomáticos cardiovasculares y, en menor frecuencia, asintomáticos respiratorios o afebriles. Siempre se debe pensar en que los patrones de enfermedad son muy parecidos a los de infarto de miocardio, miopericarditis, etc. No tiene hoy

Tabla 4 Diagnóstico diferencial entre insuficiencia cardiaca e infección por COVID-19

\begin{tabular}{|c|c|c|}
\hline & COVID-19 & Insuficiencia cardiaca \\
\hline \multicolumn{3}{|l|}{ Analítica } \\
\hline Linfopenia & +++ & - \\
\hline ProBNP & $-1+$ & +++ \\
\hline PCR & +++ & + \\
\hline D-Dímero & +++ & $-1+$ \\
\hline \multicolumn{3}{|l|}{ Pruebas de imagen } \\
\hline \multicolumn{3}{|l|}{ Radiografia Tórax } \\
\hline Infiltrados & Periféricos & Centrales, en alas de mariposa \\
\hline Hilios aumentados & $-/+*$ & +++ \\
\hline Cardiomegalia & $-*$ & + \\
\hline Derrame pleural & $-*$ & + \\
\hline TAC & $\begin{array}{l}\text { Imágenes en vidrio deslustrado de } \\
\text { predominio periférico bilaterales (al inicio } \\
\text { pueden ser unilaterales) } \\
\text { +/-Condensaciones segmentarias } \\
\text { +/- engrosamiento pleural }\end{array}$ & $\begin{array}{l}\text { Imágenes en vidrio deslustrado de } \\
\text { predominio central } \\
\text { Condensaciones de predominio central } \\
\text { Dilatación de venas pulmonares } \\
\text { Derrame pleural }\end{array}$ \\
\hline
\end{tabular}

*Puede estar presente en IC previa

Tomada de: Implicaciones de la pandemia por COVID-19 para el paciente con insuficiencia cardiaca, trasplante cardiaco y asistencia ventricular. Recomendaciones de la Asociación de Insuficiencia Cardiaca de la Sociedad Española de Cardiología. 
Tabla 5 Trasplante de corazón, inmunosupresión e infección por Covid ${ }^{4}$

\begin{tabular}{|l|l|}
\hline Enfermedad leve & $\begin{array}{l}\text { Mantener pauta habitual de inmunosupresión o } \\
\text { reducir/suspender micofenolato/azatioprina } 48 \text { h. }\end{array}$ \\
\hline Enfermedad moderada-severa & $\begin{array}{l}\text { Considerar suspender micofenolato/azatioprina y reducir } \\
\text { niveles de anticalcineurínico. Se puede aumentar la } \\
\text { corticoterapia o incluso administrar inmunoglobulinas. }\end{array}$ \\
\hline Terapia farmacológica & $\begin{array}{l}\text { No existe evidencia específica de ningún tratamiento y se } \\
\text { deben establecer protocolos en cada centro. Se han utilizado } \\
\text { cloroquina, hidroxicloroquina, inmunoglobulina endovenosa, } \\
\text { suero de pacientes con infección por COVID-19 resuelta, } \\
\text { remdesivir, esteroides a altas dosis y tocilizumab. Se } \\
\text { recomienda cautela en el uso de lopinavir/ritonavir, } \\
\text { darunavir/ritonavir y darunavir/cobicistat por las } \\
\text { interacciones farmacológicas significativas con los } \\
\text { inmunosupresores. }\end{array}$ \\
\hline
\end{tabular}

Tomada de: Implicaciones de la pandemia por COVID-19 para el paciente con insuficiencia cardiaca, trasplante cardiaco y asistencia ventricular. Recomendaciones de la Asociación de Insuficiencia Cardiaca de la Sociedad Española de Cardiología.

criterios clínicos que obliguen a la toma de prueba diagnóstica, por lo que la búsqueda exhaustiva permitirá su diagnóstico precoz.

\section{Tratamiento}

El tratamiento actual para pacientes con miocarditis aguda fulminante es el siguiente (recomendación extrapolada de hallazgos en neumonía y reporte de casos):

1. Manejo de soporte habitual de UCl (soporte ventilatorio, vasoactivo, etc.).

2. Tratamiento inotrópico (levosimendan, milrinona).

3. Toma de electrocardiograma inicial; registrar PR y QT c inicial.

4. Administración de hidrocloroquina $400 \mathrm{mg}$ cada 12 horas día 1, $200 \mathrm{mg}$ cada 12 horas hasta completar 7 días.

5. Administración de azitromicina $500 \mathrm{mg}$ diarios, hasta completar 6-7 días (estricta vigilancia del intervalo QT en el electrocardiograma diario).

6. Si QTC mayor a 500, considerar drogas como betabloqueadores y magnesio para reducirlo evitando al máximo la retirada de la medicación antiviral.

7. Administración de lopinavir/ritonavir 400/100 vía oral cada 12 horas por 10 días.

8. Si requiere profilaxis gástrica, evitar antihistamínicos como ranitidina.

9. Inicio de anticoagulación parenteral en caso de aumento del dímero $\mathrm{D}$.

10. Inicio de esteroides IV metilprednisolona $200 \mathrm{mg}$ IV diarios $\mathrm{x} 4$ días $\mathrm{y} / \mathrm{o}$ en conjunto con inmunoglobulina humana $20 \mathrm{~g}$ día $x 4$ días (datos extrapolados de casos de reporte con recuperación exitosa); no tiene indicación INVIMA para este fin y es preciso considerar los recursos del hospital.

\section{Paciente con trasplante cardiaco}

Se recomiendan medidas estrictas de aislamiento y visitas rutinarias vía telefónica. Se desaconseja la realización de biopsias endomiocárdicas y ecocardiogramas de control para evitar el contacto con el hospital (solo durante los tres primeros meses o tras un episodio de rechazo).

\section{Paciente con trasplante cardiaco y sospecha/confirmación de infección por COVID-19}

Se recomienda hacer TAC de tórax a los pacientes con trasplante cardiaco y síntomas respiratorios. En cuanto al manejo terapéutico se recomienda:

1. Administración de hidrocloroquina $400 \mathrm{mg}$ cada 12 horas día $1,200 \mathrm{mg}$ cada 12 horas hasta completar 7 días.

2. Administración de azitromicina $500 \mathrm{mg}$ diarios hasta completar 6-7 días (estricta vigilancia del intervalo QT en el electrocardiograma diario).

3. Si QTC mayor a 500, considerar drogas como betabloqueadores y magnesio para reducirlo evitando al máximo la retirada de la medicación antiviral.

4. Administración de lopinavir/ritonavir 400/100 vía oral cada 12 horas por 10 días.

5. Si requiere profilaxis gástrica, evitar antihistamínicos como ranitidina.

6. Inicio de anticoagulación parenteral en caso de aumento del dímero $\mathrm{D}$ elevado.

\section{Reto terapéutico en inmunosupresión (recomendaciones generales) (tabla 6)}

1. En caso de tener tratamiento con tacrólimus y ritonavir se plantea lo siguiente: 
Tabla 6 Propuesta de tratamiento farmacológico en Covid (estudios en curso)

\begin{tabular}{|c|c|c|}
\hline Fármaco & Dosis & $\begin{array}{l}\text { Efectos adversos más frecuentes, contraindicaciones e } \\
\text { interacciones }\end{array}$ \\
\hline Hidroxicloroquina & $\begin{array}{l}400 \text { mg cada } 12 \mathrm{~h} \text { VO } \\
\text { el primer día y } 200 \\
\text { mg cada } 12 \mathrm{~h} \\
\text { durante } 5 \text { días }\end{array}$ & $\begin{array}{l}\text { Queratopatía, prolongación del QT } \\
\text { Interacciones con fármacos que alargan QT Aumentan } \\
\text { niveles de anticalcineurínicos e inhibidores de mTOR } \\
\text { Dosis en insuficiencia renal: } \\
\text { FG } 30-50 \mathrm{ml} / \mathrm{min} \text { : administrar } 75 \% \text { de la dosis } \\
\text { FG } 10-30 \mathrm{ml} / \mathrm{min} \text { : administrar el } 50 \% \text { de la dosis } \\
\text { FG }<10 \mathrm{ml} / \mathrm{min} \text { : administrar entre el } 25-50 \% \text { de la dosis }\end{array}$ \\
\hline Cloroquina & $500 \mathrm{mg}$ cada $12 \mathrm{~h}$ & $\begin{array}{l}\text { Similares a hidroxicloroquina } \\
\text { Si FG }<10 \mathrm{ml} / \mathrm{min} \text {, disminuir dosis a } 500 \mathrm{mg} \text { cada } 24 \mathrm{~h}\end{array}$ \\
\hline Lopinavir/ritonavir & $\begin{array}{l}400 / 100 \text { mg cada } 12 \\
\text { h VO durante por } 14 \\
\text { días }\end{array}$ & $\begin{array}{l}\text { Diarrea, náuseas, vómitos, prolongación del QT } \\
\text { No ajuste en insuficiencia renal } \\
\text { Contraindicado en insuficiencia hepática grave } \\
\text { Interacciones con hipolipemiantes, fármacos que } \\
\text { alargan QT, anticoagulantes orales, anticalcineurínicos e } \\
\text { inhibidores de mTOR }\end{array}$ \\
\hline
\end{tabular}

\begin{tabular}{|c|c|c|}
\hline Darunavir/ Ritonavir & $\begin{array}{l}600 / 100 \mathrm{mg} \text { cada } 12 \\
\text { horas durante } 14 \text { días } \\
800 / 100 \mathrm{mg} \text { cada } 24 \\
\text { horas durante } 14 \text { días }\end{array}$ & $\begin{array}{l}\text { Mejor tolerancia digestiva, por el resto similar a } \\
\text { lopinavir/ritonavir }\end{array}$ \\
\hline Darunavir/cobicistat & $\begin{array}{l}800 / 125 \text { mg cada } 24 \\
\text { horas durante } 14 \text { días }\end{array}$ & $\begin{array}{l}\text { Mejor tolerancia digestiva, por el resto similar a } \\
\text { lopinavir/ritonavir } \\
\text { No recomendado en insuficiencia renal }\end{array}$ \\
\hline Interferon $\beta 1 b$ & $\begin{array}{l}0,25 \mathrm{mg} \text { cada } 48 \mathrm{~h} \mathrm{SC} \\
\text { durante } 14 \text { días }\end{array}$ & $\begin{array}{l}\text { Fiebre, cefalea, síndrome pseudogripal } \\
\text { Contraindicado en insuficiencia hepática } \\
\text { Riesgo teórico de rechazo agudo del injerto por } \\
\text { inducción de la alorreactividad }\end{array}$ \\
\hline Remdesivir & $\begin{array}{l}\text { Dosis de carga: } 200 \\
\text { mg IV por un día y } \\
100 \mathrm{mg} / 24 \text { h IV } \\
\text { durante } 9 \text { días }\end{array}$ & $\begin{array}{l}\text { Hipotensión durante la infusión } \\
\text { No se conocen las interacciones }\end{array}$ \\
\hline Tocilizumab & $\begin{array}{l}8 \mathrm{mg} / \mathrm{Kg} \text { IV (dosis } \\
\text { máxima de } 800 \mathrm{mg} \text { ) }\end{array}$ & Hipertrigliceridemia, elevación de transaminasas \\
\hline
\end{tabular}

FG: filtrado glomerular; IV: Intravenosa; SC: subcutáneo; VO: vía oral.

El remdesivir es un fármaco de uso compasivo que se puede valorar en casos graves. El tocilizumab tiene indicación basada en la experiencia de una serie pequeña de casos con síndrome de distrés respiratorio agudo grave.

Tomada de: Implicaciones de la pandemia por COVID-19 para el paciente con insuficiencia cardiaca, trasplante cardiaco y asistencia ventricular. Recomendaciones de la Asociación de Insuficiencia Cardiaca de la Sociedad Española de Cardiología.

a. Reducir dosis a 0,03-0,08 mg/kg/día con toma de niveles cada $48-72$ horas con el fin de mantener la dosis de inmunosupresión en caso de órgano sólido vital.

2. En caso de tener tratamiento con everolimus y ritonavir, diferir tratamiento dado que la interacción es potente y puede generar la aparición de úlceras severas o potenciar los efectos secundarios, al menos durante el tratamiento con el antiviral.

3. Incrementar la dosis de esteroide a 0,3 a $0,5 \mathrm{mg} / \mathrm{kg} /$ día con el fin de mantener la prevención de rechazo y vitalidad del órgano.

4. El uso de inmunoglobulina en trasplante cardiaco y situaciones similares no tiene indicación INVIMA; por tanto, su uso por fuera de este contexto requiere una evaluación crítica del grupo tratante (riesgo / beneficio).

\section{Paciente con falla cardiaca avanzada y necesidad de trasplante cardiaco urgente o ambulatorio}

Ante el contexto de pandemia se recomienda realizar una evaluación detallada del paciente para definir riesgo/beneficio de un trasplante cardiaco. En caso de que se decida realizarlo, se deben hacer estudios del donante y del receptor que muestren resultados negativos para SARSCoV2.

No se recomienda trasplante cardiaco urgente dado el mal pronóstico a corto plazo y el alto riesgo de infección por COVID-19.

En pacientes que han presentado infección por coronavirus se recomienda esperar 14 días para realizar un trasplante cardiaco $^{32}$. 


\section{Pacientes ambulatorios con asistencia ventricular de larga duración}

Este grupo es de alto riesgo debido a insuficiencia cardiaca crónica; por consiguiente, tienen mayor riesgo de infección y complicaciones.

1. Realizar aislamiento estricto y evitar visitas médicas durante la pandemia.

2. Si se dispone de dispositivo CoaguCheck ${ }^{\circledR}$ se recomienda para el seguimiento del INR.

3. Educar a todos los pacientes para que realicen la monitorización de los parámetros del dispositivo.

4. Se recomienda evaluación por vía telefónica a cargo de personal de enfermería para detectar alguna alteración que requiera valoración médica ${ }^{32}$.

\section{Manejo farmacológico para la infección por SARS-CoV2 y su impacto en el sistema cardiovascular}

En la tabla 7 se describen los medicamentos potenciales para el tratamiento por infección por SARS-CoV2 y sus posibles efectos sobre el sistema cardiovascular.

\section{Conclusión}

La situación actual de pandemia a causa del SARS-CoV2 COVID-19 es una infección de rápida diseminación en todo el mundo, que ha cobrado miles de vidas, incluyendo las del personal de salud. Se hace imperativo conocer el compromiso cardiovascular que puede presentar el paciente con esta enfermedad, desde evaluar la población a riesgo para adoptar medidas tempranas de prevención, como identificar estos pacientes al ingreso hospitalario y durante su hospitalización, pues presentan un mayor riesgo de deterioro y mortalidad. El escenario clínico puede presentarse por lesión miocárdica, insuficiencia cardiaca y arritmias. Puede ocurrir como consecuencia de un evento coronario agudo isquémico por trombosis coronaria o por alteración en la demanda y suplencia de oxígeno. Se han descrito casos de miocarditis viral fulminando con derrame pericárdico y taponamiento cardiaco, por lo cual se debe sospechar infección por COVID-19 en estos pacientes. Además, durante el tratamiento se deben tener en cuenta las comorbilidades y los posibles efectos adversos de los medicamentos para realizar vigilancia estricta e intervención adecuada. El mejor tratamiento es la prevención. Educar a los pacientes sobre la importancia del aislamiento en casa, el lavado de manos y las medidas preventivas siempre será mejor que tratar las complicaciones.

\section{Conflicto de intereses}

Ninguno.

\section{Bibliografía}

1. Wu Z, McGoogan JM. Characteristics of and important lessons from the coronavirus disease 2019 (COVID-19) Outbreak in China: Summary of a Report of 72314 Cases From the Chinese Center for Disease Control and Prevention. JAMA. 2020.

2. Huang C, Wang Y, Li X, et al. Clinical features of patients infected with 2019 novel coronavirus in Wuhan China. Lancet. 2020.

3. Driggin E, Madhavan MV, Bikdeli B, et al. Cardiovascular Considerations for Patients Health Care Workers, and Health Systems During the Coronavirus Disease 2019 (COVID-19) Pandemic. J Am Coll Cardiol. 2020, http://dx.doi.org/10.1016/j.jacc.2020.03.031.

4. Johns Hopkins. Coronavirus Resource Center Disponible en: https://coronavirus.jhu.edu/map.html.

5. Mehra MR, Ruschitzka F. COVID-19 illness and heart failure: a missing link? JACC: Heart Failure. 2020, http://dx.doi.org/10.1016/j.jchf.2020.03.004.

6. Wang D, Hu B, Hu C, et al. Clinical Characteristics of 138 hospitalized patients with 2019 novel coronavirus-infected pneumonia in Wuhan China. JAMA. 2020.

7. Bansal M. Cardiovascular disease and COVID-19, diabetes \& metabolic syndrome: Clinical Research \& Reviews. Disponible en: https://doi.org/10.1016/j.dsx.2020.03.013.

8. Lippi G, Sanchis-Gomar F, Henry BM. Coronavirus disease 2019 (COVID-19): the portrait of a perfect storm. Ann Transl Med. 2020.

9. Zhang TWQ, Zhang Z. Probable pangolin origin of SARS-CoV2 associated with the COVID-19 outbreak. Curr Biol. March. 2020;13, http://dx.doi.org/10.1016/j.cub.2020.03.022.

10. Zheng YY, Ma YT, Zhang JY, Xie X. COVID-19 and the cardiovascular system. Nature Reviews Cardiology. 2020:1-2.

11. Lippi G, Plebani M. Laboratory abnormalities in patients with COVID-2019 infection. Clin Chem Lab Med. 2020.

12. Lippi G, Plebani M. Procalcitonin in patients with severe coronavirus disease 2019 (COVID-19): a meta-analysis. Clin Chim Acta. 2020.

13. Shen Z, Xiao Y, Kang L, et al. Genomic diversity of SARS-CoV2 in coronavirus disease 2019 patients. Clin Infect Dis. 2020.

14. Li Q, Guan X, Wu P, et al. Early transmission dynamics in Wuhan China, of novel coronavirus-infected pneumonia. N Engl J Med. 2020.

15. Remuzzi A, Remuzzi G. COVID-19 and Italy: what next? Lancet. 2020.

16. Zhao S, Lin Q, Ran J, et al. Preliminary estimation of the basic reproduction number of novel coronavirus (2019- nCoV) in China, from 2019 to 2020: A data-driven analysis in the early phase of the outbreak. Int J Infect Dis. 2020.

17. Backer JA, Klinkenberg D, Wallinga J. Incubation period of 2019 novel coronavirus (2019-nCoV) infections among travelers from Wuhan China, 20-28 January 2020. Euro Surveill. 2020, doi: 10.2807/1560-7917. ES.2020.25.5.2000062.

18. Nishiura $\mathrm{H}$, Kobayashi $\mathrm{T}$, Miyama $\mathrm{T}$, et al. Estimation of the asymptomatic ratio of novel coronavirus infections (COVID-19). Int J Infect Dis. 2020.

19. Mizumoto K, Kagaya K, Zarebski A, et al. Estimating the asymptomatic proportion of coronavirus disease 2019 (COVID-19) cases on board the Diamond Princess cruise ship, Yokohama Japan, 2020. Eurosurveillance. 2020;25:2000180.

20. Wu Z, McGoogan JM. Characteristics of and important lessons from the coronavirus disease 2019 (COVID-19) outbreak in China: Summary of a Report of 72314 Cases From the Chinese Center for Disease Control and Prevention. JAMA. 2020.

21. Van Doremalen N, Bushmaker T, Morris DH, et al. Aerosol and Surface Stability of SARS-CoV2 as Compared with SARS-CoV-1. N Engl J Med. 2020.

22. CORONAVIRUS (COVID-19). Disponible en: WwW.minsalud.gov.co.

23. Giudicessi J, Noseworthy P, Friedman P, et al. Urgent guidance for navigating and circumventing the QTc prolonging and torsadogenic potential of possible pharmacotherapies for COVID-19. Mayo Clinic Proceedings March. 2020;25. 
24. Chen D, Li X, Song Q, et al. Hypokalemia and clinical implications in patients with coronavirus disease 2019 (COVID-19). medRxiv. 2020, 2020.02.27.20028530.

25. Guan WJ, Ni ZY, Hu Y, et al. Clinical characteristics of coronavirus disease 2019 in China. New Eng J Med. 2020, http: //dx.doi.org/10.1056/NEJMoa2002032.

26. Shi S, Qin M, Shen B, et al. Association of cardiac injury with mortality in hospitalized patients with COVID-19 in Wuhan China. 2020.

27. Hu H, Ma F, Wei X, et al. Coronavirus fulminant myocarditis saved with glucocorticoid and human inmunoglobulin Cardiovascular Flashlight. Eur Heart J. 2020. Mar 16.

28. Hua A, O'Gallagher K, Sado D, et al. Life-threatening cardiac tamponade complicating myo-pericarditis in COVID-19 Cardiovascular Flashlight. Eur Heart J. 2020. Apr 1.
29. Liu K, Fang YY, Deng Y, et al. Clinical characteristics of novel coronavirus cases in tertiary hospitals in Hubei province. Chin Med J (Engl). 2020.

30. Tian S, Xiong Y, Liu H, et al. Pathological study of the 2019 novel coronavirus disease (COVID-19) through post-mortem core biopsies. Preprints. 2020, 2020030311, doi: 10.20944/preprints202003.0311.v1.

31. Wu Q, Zhou L, Sun X, et al. Altered lipid metabolism in recovered SARS patients twelve years after infection. Sci Rep. 2017;7:9110.

32. Implicaciones de la pandemia por COVID-19 para el paciente con insuficiencia cardiaca, trasplante cardiaco y asistencia ventricular. Recomendaciones de la Asociación de Insuficiencia Cardiaca de la Sociedad Española de Cardiología. 